\title{
THE DISTRIBUTION OF INTEGERS REPRESENTED BY BINARY QUADRATIC FORMS
}

\section{GORDON PALL}

R. D. James ${ }^{1}$ has proved the following theorem:

THEOREM 1. Let $B(x)$ denote the number of positive integers $m \leqq x$ which can be represented by positive, primitive, binary quadratic forms of a given negative discriminant $d$, but are prime to $d$. Then

$$
B(x)=b x /(\log x)^{1 / 2}+O(x / \log x),
$$

where $b$ is the positive constant given by

$$
\pi b^{2}=\prod_{q}\left(1-1 / q^{2}\right)^{-1} \prod_{p \mid d}(1-1 / p) \sum_{n=1}^{\infty}(d \mid n) n^{-1} .
$$

Here $q$ runs over all primes such that $(d \mid q)=-1 ; p$ denotes any prime greater than or equal to 2 ; and $(d \mid n)$ is the Kronecker symbol.

We shall deduce from his result an asymptotic formula with the restriction that $m$ be prime to $d$ removed.

First, let $p$ be a prime dividing $d$ but not satisfying

$$
p>2 \text { and } p^{2} \mid d \text {, or } p=2 \text { and } d \equiv 0 \text { or } 4(\bmod 16) \text {. }
$$

Then $p n$ is represented by p. p. b. q. forms of discriminant $d$ if and only if $n$ is likewise represented. ${ }^{2}$ Hence if $p^{r} \leqq(\log x)^{1 / 2}$ then the number of represented integers less than or equal to $x$ of the form $p^{r} m$ with $m$ prime to $d$, is

$$
\frac{b x / p^{r}}{\left(\log x / p^{r}\right)^{1 / 2}}+O\left(\frac{x / p^{r}}{\left(\log x / p^{r}\right)}\right)=\frac{b x / p^{r}}{(\log x)^{1 / 2}}+O\left(\frac{x / p^{r}}{\log x}\right)
$$

since $\left(\log x p^{-r}\right)^{-1 / 2}-(\log x)^{-1 / 2}=O\left(\left(\log p^{r}\right) /(\log x)^{3 / 2}\right)$. Also, if $p^{8}>(\log x)^{1 / 2}$,

$$
\left(b x /(\log x)^{1 / 2}\right)\left(1 / p^{s}+1 / p^{s+1}+\cdots\right)=O(x /(\log x)) .
$$

Hence the number of positive integers less than or equal to $x$, represented by p.p.b.q. forms of discriminant $d$, and prime to $d$ except that they need not be prime to $p$, is given by 1942 .

Presented to the Society, September 10,1942; received by the editors August 4,

${ }^{1}$ R. D. James, Amer. J. Math. vol. 60 (1938) pp. 737-744.

${ }^{2}$ G. Pall, Math. Zeit. vol. 36 (1933) pp. 321-343, p. 331. 


$$
\frac{b x}{(\log x)^{1 / 2}}\left(1+1 / p+\cdots+1 / p^{r}+\cdots\right)+O(x / \log x) .
$$

Thus each prime $p$ dividing $d$ but not satisfying (3) can be removed from the set of primes excluded in $m$, by replacing $b$ by $b /(1-1 / p)$. We recall that ${ }^{3}$

$$
\sum(d \mid n) n^{-1}=2 \pi h(d) /\left(w(-d)^{1 / 2}\right),
$$

where $h(d)$ is the number of p.p.b.q. classes of discriminant $d$, and $w=2$, except that $w=4$ if $d=-4, w=6$ if $d=-3$. Hence the number of positive integers less than or equal to $x$ which can be represented by p.p.b.q. forms of discriminant $d$, and which have no prime factor $p$ satisfying (3), is

$$
b_{0} x /(\log x)^{1 / 2}+O(x / \log x)
$$

where

$$
\begin{aligned}
b_{0}^{2}=\left(2 h(d) / w(-d)^{1 / 2}\right) & \prod_{q}\left(1-1 / q^{2}\right)^{-1} \\
& \prod_{p \text { sat. (3) }}(1-1 / p) \prod_{p \mid d, \text { not (3) }}(1-1 / p)^{-1} .
\end{aligned}
$$

Second let $p$ satisfy (3). Then ${ }^{4} p m$ is not represented by p.p.b.q. forms of discriminant $d$ if $p \nmid m$; and $p^{2} n$ is represented by such forms if and only if $n$ is represented by p.p.b.q. forms of discriminant $d_{1}=d / p^{2}$. If $b_{1}$ is the value of $b_{0}$ corresponding to $d_{1}$, then from (5),

$$
\begin{aligned}
b_{1} & =b_{0} & & \text { if } d_{1} / p^{2} \text { is an integer } \equiv 0 \text { or } 1 \bmod 4, \\
& =b_{0}\left(1-p^{-2}\right)^{-1} & & \text { if }\left(d_{1} \mid p\right)=-1, \\
& =b_{0}\left(1-p^{-1}\right)^{-1} & & \text { otherwise. }
\end{aligned}
$$

Hence if $d=p^{2 k} d^{\prime}$, where $k \geqq 1$ and $p^{2} \nmid d^{\prime}$, we can remove $p$ from the set of excluded primes by replacing $b_{0} x /(\log x)^{1 / 2}$ by $\left(b_{0} x /(\log x)^{1 / 2}\right)$ $\left(1+p^{-2}+\cdots+p^{-2 k+2}+p^{-2 k} \lambda\right)$, where $\lambda=1 /(1-1 / p)$ if $\left(d^{\prime} \mid p\right) \neq-1$, $\lambda=1 /\left(1-1 / p^{2}\right)$ if $\left(d^{\prime} \mid p\right)=-1$; hence by multiplying $b_{0}$ by

$$
\frac{1+p^{-2 k-1}}{1-p^{-2}} \text { if }\left(d^{\prime} \mid p\right) \neq-1, \quad \frac{1}{1-p^{-2}} \text { if }\left(d^{\prime} \mid p\right)=-1
$$

Thus we have finally this theorem:

THEOREM 2. The number $C(x)$ of positive integers $n \leqq x$ which can be

${ }^{3}$ See, for example, Landau, Vorlesungen ïber Zahlentheorie, vol. 2, p. 152.

${ }^{4}$ G. Pall, Amer. J. Math. vol. 57 (1935) pp. 789-799, formula (9). 
represented by positive, primitive, binary quadratic forms of a given negative discriminant $d$ is given by

$$
c x /(\log x)^{1 / 2}+O(x / \log x),
$$

where $c$ is the positive constant defined by

$$
c=b_{0} \prod_{p \text { sat. (3) }}\left(1-p^{-2}\right)^{-1} \cdot \prod\left(1+p^{-2 k-1}\right),
$$

where in the last product $d=p^{2 k} d^{\prime}$, where $p^{2} \nmid d^{\prime}, k \geqq 1$, and $\left(d^{\prime} \mid p\right) \neq-1$.

Example. If $d=-3$,

$$
\begin{aligned}
c^{2}=b_{0}^{2} & =3^{-1} \cdot 3^{-1 / 2} \cdot \alpha \cdot(3 / 2), & \alpha & =\prod_{q}^{q \equiv 2(3)}\left(1-q^{-2}\right)^{-1}, \\
& =\alpha /\left(2(3)^{1 / 2}\right), & c & =.64 \text { approximately. }
\end{aligned}
$$

If $d=-12, b_{0}^{2}=\left(1 /(12)^{1 / 2}\right) \prod_{q(\neq 2)}^{\prime}\left(1-q^{-2}\right)^{-1} \cdot(1 / 2) \cdot(3 / 2)=9 \alpha /\left(32(3)^{1 / 2}\right)$; and by $(7), c^{2}=b_{0}^{2}(16 / 9)=\alpha /\left(2(3)^{1 / 2}\right)$. Hence $c$ is the same for $d=-12$ as for $d=-3$. This agrees with the fact that $x^{2}+3 y^{2}$ represents exactly the same numbers as $x^{2}+x y+y^{2}$.

MCGILl UNIVERSITY 